\title{
Esquema de vacunación para el grupo de edad de 0-18 años
}

\author{
Oscar Porras, Arturo Abdelnour, Efraín Artavia, Aristides Baltodano, \\ Mario Barrantes, Julia Fernandez, Ramón Rivera
}

El esquema de vacunación es una recomendación basada en evidencia, que permite a una población decidir la forma en que puede prevenir, en diferentes grupos de edad, enfermedades transmisibles por medio de la inmunización de sus habitantes. La estrategia y programas de vacunación de un país deben responder a un plan nacional de salud, basado en el concepto de que la prevención siempre es costo-efectiva.

La vacunación de niños y adolescentes ha demostrado ser costo efectiva en todos los grupos humanos. En especial en este segmento de edad, ha demostrado su efectividad como medida para reducir la mortalidad infantil, la morbilidad y los efectos de las enfermedades infecciosas que producen minusvalía.

Costa Rica ha experimentado, durante los últimos años, la aparición de diferencias en las oportunidades de prevención con inmunizaciones, en los diferentes estratos sociales de su población. Las opciones mínimas las ofrece el sistema de salud estatal, un segmento de la población al cual se podría ampliar este esquema agregando dos opciones de vacunación y un pequeño grupo social que puede ofrecer a sus hijos el esquema disponible completo.

En este documento la Asociación Costarricense de Pediatría (ACOPE), desea ofrecer a los padres y madres, a los profesionales en salud y a las autoridades una visión de lo que debe ser el esquema de inmunizaciones en la población costarricense de 0-18 años. ACOPE no acepta esquemas basados en la capacidad económica de los diferentes estratos sociales, debe haber una estrategia nacional de vacunación y el estado ser responsable de ofrecer el esquema de inmunizaciones óptimo a todos los niños (as) y adolescentes que viven en el territorio nacional.

ACOPE va a ofrecer revisiones anuales del esquema de vacunación para mantenerlo actualizado con nuevas vacunas, formulaciones y cambios en la edad de aplicación.

Asociación Costarricense de Pediatría

Abreviaturas: ACOPE, Asociación Costarricense de Pediatría,

\section{Correspondencia:}

Dr. Oscar Porras Madrigal (PhD)

Servicio de Inmunología y

Reumatología Pediátrica

Hospital Nacional de Niños

"Dr. Carlos Sáenz Herrera"

Apartado 16541000 San José,

Costa Rica

Fax: 2235125

Email:porrasza@racsa.co.cr

ISSN 0001-6002/2006/48/4/204-207 Acta Médica Costarricense, (c)2006

Colegio de Médicos y Cirujanos

\section{El esquema de vacunación}

El esquema de vacunación es una guía de inmunizaciones, técnicamente diseñada, que indica, para las vacunas aprobadas en un país, cuáles son las edades de aplicación, el número de dosis, la vía de aplicación y la cantidad de vacuna por dosis.

En el cuadro 1 se indica la distribución de las diferentes vacunas de acuerdo a la edad del niño (a) o adolescente y el número de dosis que se deben aplicar en el grupo de edad de 0-18 años.

En el cuadro 2 se anotan las diferencias principales entre el esquema oficial de vacunación y el esquema disponible a nivel nacional.

Descriptores: vacunas, inmunizaciones, esquemas de vacunación 


\begin{tabular}{|c|c|c|c|c|c|c|c|c|c|c|c|c|c|}
\hline \multicolumn{14}{|c|}{$\begin{array}{l}\text { Cuadro 1. Asociación Costarricense de Pediatría } \\
\text { Esquema de vacunación para el grupo de edad de 0-18 años de edad }\end{array}$} \\
\hline $\begin{array}{l}\text { Edad } \\
\text { Vacuna }\end{array}$ & $\begin{array}{l}\text { Recién } \\
\text { Nacido }\end{array}$ & $\begin{array}{l}2 \\
\text { meses }\end{array}$ & $\begin{array}{l}4 \\
\text { meses }\end{array}$ & $\begin{array}{l}6 \\
\text { meses }\end{array}$ & $\begin{array}{l}12 \\
\text { meses }\end{array}$ & $\begin{array}{l}15-18 \\
\text { meses }\end{array}$ & $\begin{array}{l}23 \\
\text { meses }\end{array}$ & $\begin{array}{l}24 \\
\text { meses }\end{array}$ & $\begin{array}{l}4 \\
\text { años }\end{array}$ & $\begin{array}{l}6 \\
\text { años }\end{array}$ & $\begin{array}{l}9 \\
\text { años }\end{array}$ & $\begin{array}{l}15 \\
\text { años }\end{array}$ & $\begin{array}{l}18 \\
\text { años }\end{array}$ \\
\hline \multicolumn{14}{|l|}{ BCG } \\
\hline \multicolumn{14}{|l|}{ Hepatitis B } \\
\hline \multicolumn{14}{|c|}{$\begin{array}{l}\text { DPT (P: célula completa } \\
\text { o Pa: acelular) }\end{array}$} \\
\hline \multicolumn{14}{|l|}{$d T^{*}$} \\
\hline \multicolumn{14}{|c|}{$\begin{array}{l}\text { Haemophilus influenzae } \\
b \text { Conjugada }\end{array}$} \\
\hline \multicolumn{14}{|c|}{$\begin{array}{l}\text { Streptococcus } \\
\text { pneumoniae Conjugada }\end{array}$} \\
\hline \multicolumn{14}{|c|}{$\begin{array}{l}\text { Polio (virus vivos vo o } \\
\text { muertos sc) }\end{array}$} \\
\hline \multicolumn{14}{|c|}{$\begin{array}{l}\text { Sarampion-rubeola- } \\
\text { paperas }\end{array}$} \\
\hline Varicela & & & & & \multicolumn{9}{|c|}{12 meses a 13 años: 1 dosis // >13 años: 2 dosis separadas 4-8 semanas } \\
\hline Hepatitis a & & & & & 2 dosis $s$ & eparadas & 6 meses & & & & & & \\
\hline Virus influenza & & & & 1 dosis & anual a pó & artir de lo: & s 6 mese & de edad & & & & & \\
\hline
\end{tabular}

*Cuando este disponible en el país dPaT (vacuna con componente acelular de tosferina), se debe recomendar su uso con el objetivo de evitar tosferina en adultos y prevenir la transmisión de Tosferina de adultos a recién nacidos y lactantes.

\section{Cuadro 2. Diferencias en las vacunas aplicadas por los proveedores.}

\begin{tabular}{|lll|}
\hline VACUNA & SISTEMA ESTATAL & SISTEMA AMPLIADO \\
\hline Difteria-Tosferina-Tétanos & Pertusis & Pertusis acelular \\
& célula completa & $3-5$ componentes \\
Polio & Virus vivos atenuados vo & Virus muertos parenteral im \\
Conjugada 7V contra Pneumococo & No & Si \\
Varicela & No & Si \\
Hepatitis A & No & Si \\
Pentavalente & Si & Si \\
Hexavalente & No & Si \\
\hline
\end{tabular}

\section{Notas específicas sobre las vacunas}

1. Difteria-Tosferina-Tétanos: esta vacuna ofrece diferen-tes opciones: para diferentes grupos de edad, componentes de Tosferina y combinadas con Polio, H. influenzae b, Hepatitis B.

a. Tosferina (Pertusis) se ofrecen dos opciones, las vacunas de célula completa (Bordetella pertussis) y las acelulares (combinaciones de 3-5 componentes).
Ambas presentaciones son semejantes en relación con la frecuencia de efectos adversos mayores y en inmunogenicidad. Sin embargo ACOPE recomienda dar preferencia a las presentaciones de vacuna acelular porque tienen la menor frecuencia de efectos adversos menores. Además, dado que la vacuna acelular es la única aprobada en adolescentes, es importante uniformar el tipo de vacuna con el fin de disminuir los errores potenciales que pueden ocurrir al mezclar diferentes tipos de vacunas. 
b. Difteria: el toxoide tiene 2 presentaciones, una con para niños (as) (DPT) y otra que se usa en adultos (dT). Además se ofrece una presentación: dPaT, para incluir el refuerzo de la vacunación contra Tosferina en la estrategia de refuerzos contra Difteria y Tétanos para adolescentes y adultos.

c. Refuerzos: los refuerzos de la vacunación básica de cuatro dosis se deben aplicar como dT o dPaT a los 9 años (5 años después de la cuarta dosis) y luego cada 10 años con dT.

d. dPaT se recomienda en adolescentes de 11-12 años que completaron el esquema básico pero que no han recibido un refuerzo con dT. Sin embargo esta vacuna no está disponible en Costa Rica. En adolescentes de 13-18 años que no han recibido que no recibieron el refuerzo de $\mathrm{dT}$ a los 9 años, se recomienda que reciban una dosis de $\mathrm{dPaT}$. Los refuerzos siguientes se deben realizar cada 10 años con dT.

\section{S. pneumoniae (Pneumococo):}

a. Polisacáridos 7 valente conjugada: Se recomienda su uso en el grupo de edad de 0-18 meses. El uso de esta vacuna debe ser una recomendación de los médicos a todos los padres y madres de familia, para prevenir la enfermedad invasora por neumococo en la población < 4 años. La vacuna conjugada, si no se utiliza en el esquema básico, se debe utilizar de acuerdo a la edad en que se inicia la aplicación, en la siguiente forma: 7-11 meses 3 dosis 1 cada 4 semanas y 1 al año de edad, 12-23 meses 2 dosis separadas por 2 meses.

b. Polisacáridos 23 valente no conjugada: se recomienda en casos dentro de los grupos de riesgo y $>3$ años.

3. Polio: se prefiere el uso de la vacuna de virus muertos de aplicación parenteral sobre la formulación de virus vivos de uso oral. La formulación parenteral es parte de las vacunas combinadas y está disponible también como dosis única. Ambas formulaciones son aceptables en el esquema de vacunación y su efecto de protección contra polio es el mismo. El uso de la vacuna de aplicación parenteral con virus de Polio muertos evita la posibilidad de casos de Polio asociado a la vacuna y contribuye eficientemente a erradicar el virus de Polio de Costa Rica.

4. Vacunas combinadas: son efectivas, inmunogénicas y representan una facilidad en su aplicación y una reducción significativa en el número de inyecciones que el niño o adolescente recibe. La discusión sobre su uso enfatiza la necesidad de aplicar 3 dosis en el primer año de vida y un refuerzo a los 18 meses de edad.
5. Hepatitis B: Los recién nacidos solo pueden recibir la vacuna en su presentación monovalente. Para las siguientes dosis (2 y 6 meses), se pueden utilizar vacunas combinadas. Los hijos (as) de madres con HBsAg positivo, deben recibir el esquema de vacunación y además $0,5 \mathrm{ml}$ de Globulina hiperinmune contra Hepatitis B dentro de las primeras 12 horas de vida. La vacuna y la inmunoglobulina deben aplicarse en sitios diferentes. Se permite administrar cuatro dosis cuando se usan vacunas combinadas; si se usa vacuna monovalente no se requiere una dosis a los 4 meses de edad. La evaluación serológica para Hepatitis B en niños (as) nacidos de madres positivas para HbsAg se debe realizar a los 9-18 meses de edad.

6. Meningococo: en Costa Rica no hay una vacuna conjugada contra Meningococo, el uso de vacunación en casos de brotes o epidemia debe seguir las indicaciones basadas en la cepa aislada, que den las autoridades de salud de Costa Rica. La vacunación contra Meningococo no es parte el esquema básico de vacunación de niños (as) y adolescentes.

7. Rotavirus: al momento de la emisión de estas recomendaciones no esta disponible en el mercado nacional una vacuna contra Rotavirus. Los estudios publicados indican que esta va a ser una vacuna efectiva para disminuir la gravedad de los casos y la hospitalización por diarrea producida por Rotavirus.

\section{Conservación y manejo de vacunas}

Es importante que los padres, madres y profesionales manejen la información adecuada sobre la conservación de las vacunas, para garantizar la aplicación de un producto que tiene las condiciones de calidad para producir el estímulo sobre el sistema inmune y así permitir una adecuada protección contra las enfermedades prevenibles por vacunación.

El cuidado de la cadena de frío, la temperatura de conservación y la fecha de vencimiento son elementos que el vacunador debe controlar y mantener dentro de las recomendaciones para cada vacuna. El transporte de la farmacia o de la casa al consultorio médico se debe realizar conservando adecuadamente la cadena de frío.

El cuadro 3 resume las condiciones fundamentales para la óptima conservación de las vacunas.

En el cuadro 4, se anotan la vía de aplicación y dosis para cada una de las vacunas. 


\begin{tabular}{|c|c|}
\hline \multicolumn{2}{|c|}{ Cuadro 3. Almacenamiento de la vacunas. } \\
\hline Vacuna & Almacenamiento en el consultorio \\
\hline BCG & $2-8^{\circ} \mathrm{C} /$ No congelar / Proteger de la luz \\
\hline DPT / dT & $2-8^{\circ} \mathrm{C} /$ No congelar \\
\hline H. influenzae b conjugada & $2-8^{\circ} \mathrm{C} /$ No congelar \\
\hline Hepatitis B & $2-8^{\circ} \mathrm{C} /$ No congelar / Proteger de la luz \\
\hline Sarampión-Rubeola-Paperas & $2-8^{\circ} \mathrm{C} /$ No congelar / Proteger de la luz \\
\hline Varicela & $2-8^{\circ} \mathrm{C} /$ No congelar / Proteger de la luz \\
\hline Influenza & $2-8^{\circ} \mathrm{C} /$ No congelar / Proteger de la luz \\
\hline Polio virus vivos & $2-8^{\circ} \mathrm{C} /$ No congelar / Descartar después de 8 horas de abierta \\
\hline Polio virus muertos & $2-8^{\circ} \mathrm{C} / \mathrm{No}$ congelar \\
\hline Hepatitis A & $2-8^{\circ} \mathrm{C} / \mathrm{No}$ congelar \\
\hline S. pneumoniae $7 \mathrm{v}$ conjugada & $2-8^{\circ} \mathrm{C} / \mathrm{No}$ congelar \\
\hline S. pneumoniae $23 \mathrm{v}$ polisacáridos & $2-8^{\circ} \mathrm{C} /$ No congelar \\
\hline
\end{tabular}

\begin{tabular}{|lll|}
\hline \multicolumn{3}{|c|}{ Cuadro 4. Dosis y vía de aplicación. } \\
\hline VACUNA & VIA & DOSIS (ml) \\
\hline BCG & i.d & 0,1 \\
DPT / dT & i.m & 0,5 \\
H. influenzae b conjugada & i.m & 0,5 \\
Hepatitis B (AgsHB niños 10 $\mu$ g) & i.m & 0,5 \\
Sarampión-Rubeola-Paperas & s.c & 0,5 \\
Varicela & s.c & 0,5 \\
Influenza & i.m & 0,5 \\
Polio virus vivos & v.o & $2-3$ gotas \\
Polio virus muertos & i.m & 0,5 \\
Hepatitis A & i.m & 0,5 \\
S. pneumoniae 7v conjugada & i.m & 0,5 \\
S. pneumoniae 23v polisacáridos & i.m & 0,5 \\
& & \\
\hline
\end{tabular}

\section{Referencias}

Centers for Disease Control and Prevention. Recommended childhood and adolescent immunization schedule - United States, 2006. MMWR 2005; 54: q1-q4.

2. Centers for Disease Control and Prevention. Preventing tetanus, diphteria, and pertussis among adolescents: use of tetanus toxoid, reduced diphteria toxoid and acellular pertussis vaccines: recommendations of the Advisory Committee on Immunization Practices (ACIP). MMWR 2006; 55:1-44.

3. Mink CA. Introducction of tetanus toxoid and reduced diphteria toxoid vaccines in the United States. Pediatr Infect Dis J 2006; 25:363364.

4. Louie JK, Schechter R, Honarmand S, Guevara HF, Shoemaker TR, Madrigal NY y col. Severe pediatric influenzae in California, 20032005: implications for immunization recommendations. Pediatrics 2006: 117:610-618.

5. Heath PT, Bonhoeffer J. Update on new vaccines and immunisation strategies. Curr Pediatr 2006; 16:1-7.

6. Las vacunas. Porras O. Rev Méd Hosp Nal Niños Costa Rica 2004; 39:79-89. 